\title{
The Endocannabinoid System Alleviates Pain in a Murine Model of Cancer-Induced Bone Pain
}

\author{
A.L. Thompson, S.A. Grenald, H.A. Ciccone, N. BassiriRad, M.J. Niphakis, B.F. Cravatt, \\ T.M. Largent-Milnes, and T.W. Vanderah \\ Department of Medical Pharmacology, College of Medicine, University of Arizona, Tucson, Arizona (A.L.T., S.A.G., H.A.C., N.B., \\ T.M.L.-M., T.W.V); Division of Pain Medicine, Department of Anesthesiology and Critical Care Medicine, Johns Hopkins \\ University, Baltimore, Maryland (S.A.G.); and The Skaggs Institute for Chemical Biology and Department of Chemical Physiology, \\ The Scripps Research Institute, La Jolla, California (M.J.N., B.F.C.)
}

Received September 27, 2019; accepted February 10, 2020

\begin{abstract}
Metastatic breast cancer is prevalent worldwide, and one of the most common sites of metastasis is long bones. Of patients with disease, the major symptom is pain, yet current medications fail to adequately result in analgesic efficacy and present major undesirable adverse effects. In our study, we investigate the potential of a novel monoacylglycerol lipase (MAGL) inhibitor, MJN110, in a murine model of cancer-induced bone pain. Literature has previously demonstrated that MAGL inhibitors function to increase the endogenous concentrations of 2-arachydonylglycerol, which then activates $\mathrm{CB} 1$ and $\mathrm{CB} 2$ receptors to inhibit inflammation and pain. We demonstrate that administration of MJN110 significantly and dose dependently alleviates spontaneous pain behavior during acute administration compared with vehicle control. In addition, MJN110 maintains its efficacy in a chronicdosing paradigm over the course of 7 days without signs of receptor sensitization. In vitro analysis of MJN110 demonstrated a dose-dependent and significant decrease in cell viability and
\end{abstract}

proliferation of 66.1 breast adenocarcinoma cells to a greater extent than KML29, an alternate MAGL inhibitor, or the CB2 agonist JWH015. Chronic administration of the compound did not appear to affect tumor burden, as evidenced by radiograph or histologic analysis. Together, these data support the application for MJN110 as a novel therapeutic for cancer-induced bone pain.

\section{SIGNIFICANCE STATEMENT}

Current standard of care for metastatic breast cancer pain is opioid-based therapies with adjunctive chemotherapy, which have highly addictive and other deleterious side effects. The need for effective, non-opioid-based therapies is essential, and harnessing the endogenous cannabinoid system is proving to be a new target to treat various types of pain conditions. We present a novel drug targeting the endogenous cannabinoid system that is effective at reducing pain in a mouse model of metastatic breast cancer to bone.

\section{Introduction}

Multiple types of malignant tumors preferentially metastasize to bone, including sarcomas and carcinomas of the lung, breast, prostate, kidney, and thyroid (Luger et al., 2001; Coleman, 2006). The most commonly reported symptom of cancer metastasis to bone is pain (Luger et al., 2005). These tumors can display either an osteolytic, osteoblastic, or mixed

This work was supported by the National Institutes of Health National Cancer Institute [R01-CA142115], the National Institutes of Health National Institute on Drug Abuse [P01-DA041307] and by the Comprehensive Pain and Addiction- Center (CPA-C), University of Arizona Health Sciences.

Some data and figures presented in this manuscript were previously presented in an abstract at Society for Neuroscience 2018 in San Diego, CA. Presentation Number 479.02; Abstract Number 7698

https://doi.org/10.1124/jpet.119.262337. phenotype. Local destruction of the bone by the tumor causes severe, chronic pain that leads to secondary fractures and/or hypercalcemia. The resulting persistent pain state that arises from destruction of the tumor-bearing bone significantly reduces the functional status of the patient, decreases quality of life (Jimenez-Andrade et al., 2010), and is associated with increases in morbidity and mortality.

Currently, the treatment of bone cancer pain is multidisciplinary because it is difficult to manage and displays aspects of inflammatory and neuropathic pain. In addition to treating the tumor burden with radiation, hormonal, biological, and bisphosphonate adjuvant therapies, the pain is managed using analgesics (Mercadante and Fulfaro, 2007). Analgesic therapy is stratified by the severity of the pain and prevalence of breakthrough pain and can include nonsteroidal

ABBREVIATIONS: 2-AG, 2-archyidonoylglycerol; AA, arachidonic acid; CB1, cannabinoid receptor 1; CB2R, CB2 receptor; CIBP, cancer-induced bone pain; eCB, endocannabinoid; JWH015, (2-Methyl-1-propyl-1H-indol-3-yl)-1-naphthalenylmethanone; KML29, 1,1,1,3,3,3-hexafluoropropan-2-yl 4-(bis(benzo[d][1,3]dioxol-5-yl)(hydroxy)methyl)piperidine-1-carboxylate; MAGL, monoacylgylcerol lipase; MJN110, 2,5dioxopyrrolidin-1-yl 4-(bis(4-chlorophenyl)methyl)piperazine-1-carboxylate; NSAID, nonsteroidal anti-inflammatory drug; SR141716A, $\mathrm{N}$-(piperidin-1-yl)-5-(4-chlorophenyl)-1-(2,4-dichlorophenyl)-4-methyl-1H-pyrazole-3-carboxamide-HCl; SR144528, 5-(4-chloro-3-methylphenyl)-1[(4-methylphenyl)methyl]-N-[(1S,2S,4R)-1,3,3-rimethylbicyclo [2.2.1] hept-2-yl]-1H-pyrazole-3-carboxamide] XTT - 2,3-bis-(2-methoxy-4-nitro-5sulfophenyl)-2H-tetrazolium-5-carboxanilide. 
anti-inflammatory drug (NSAID) and opiate therapies, both of which have downsides. NSAID therapies are effective against mild to moderate pain and target only the inflammatory pain of the cancer. As the disease progresses, these become ineffectual. In addition, chronic therapy can increase risk of gastritis, ulcers, renal dysfunction, and cardiovascular events (Jin, 2015). Opiate therapies, although effective for the resolution of acute, severe pain when used chronically, display a wide array of severe side effects, including constipation, sedation, respiratory depression, tolerance, paradoxical hyperalgesia, and addiction (Vanderah et al., 2000). Additionally, preclinical models have demonstrated that chronic morphine treatment of animal cancer models accelerates bone loss and increases the risk of fracture compared with non-opiate-treated controls (King et al., 2007; Lozano-Ondoua et al., 2013a).

Cannabinoid compounds have been demonstrated to act as potent analgesics in models of acute, chronic, and neuropathic pain (Malan et al., 2001; Ibrahim et al., 2005, 2006; Whiteside et al., 2007). These compounds activate the CB1 receptor, the CB2 receptor (CB2R), or a combination of both. CB2R agonists produce both anti-inflammatory and antinociceptive effects (Lozano-Ondoua et al., 2010, 2013a). Recently, CB2R signaling was demonstrated to have positive effects on bone mineral density, which makes this receptor a desirable target for patients with bone cancer pain (Ofek et al., 2006; LozanoOndoua et al., 2013a). Studies with CB2R-deficient mice demonstrated enhanced loss of trabecular bone, cortical thinning, and an osteoporotic phenotype (Ofek et al., 2006), providing evidence for the endogenous cannabinoid system in the development and maintenance of the skeletal system.

CB1 and CB2 receptor activation by endogenous cannabinoids is well described. The most-studied endocannabinoids $(\mathrm{eCBs})$ are those found in the highest concentration in the human body: anandamide and 2-archyidonoylglycerol (2-AG). 2-AG is synthesized by phospholipase $\mathrm{C}$ and diacylglycerol lipase, which are found tethered to the intracellular side of the plasma membrane on postsynaptic terminals and can act as a retrograde signal (Ohno-Shosaku et al., 2012). 2-AG is degraded by the enzyme monoacylglycerol lipase (MAGL) into arachidonic acid (AA) and glycerol (Dinh et al., 2002, 2004), in which AA is able to enter the pathways leading to synthesis of inflammatory prostaglandins. The pharmacological inhibition of enzymes responsible for the degradation of $\mathrm{eCB}$, thereby increasing the amount of eCBs, have been shown to be effective in inhibiting thermal, chemical, and neuropathic pain (Hohmann et al., 2005; Suplita et al., 2005; Wilkerson et al., 2016; Curry et al., 2018). MAGL is overexpressed in many human cancer cells, and its expression is associated with increased metastatic potential and invasiveness (Kohnz and Nomura, 2014). Taken together, the inhibition of MAGL has been suggested to both inhibit pain and promote bone integrity while lowering the pathogenicity of solid tumors (Nomura et al., 2010; Hanlon et al., 2016).

In this study, we demonstrate that inhibition of MAGL has viable therapeutic application in alleviating cancer-induced bone pain (CIBP) in a murine model of metastatic breast cancer. The inhibition of MAGL significantly decreases spontaneous pain behaviors and simultaneously decreases the in vitro cancer cell viability. Together, our data suggest that MAGL inhibition may be an effective strategy to inhibit the multimodal bone pain associated with metastatic cancer.

\section{Materials and Methods}

\section{In Vivo}

Animals. All procedures were approved by the University of Arizona Animal Care and Use Committee and conformed to the Guidelines for the Care and Use of Laboratory Animals of the National Institutes of Health and the International Association for the Study of Pain. Female BALB/cAnNCrl mice aged 7-9 weeks and weighing between 15 and $20 \mathrm{~g}$ at the time of testing ( $n=8-12$ animals per treatment group after exclusion) were obtained from Charles River (Willmington, ME). All animals were maintained on a 12-hour light/ dark cycle in a climate-controlled room and were provided access to Teklad NIH-31 (\#7913; Envigo, Madison, WI) and water ad libitum. Animals were housed in standard cages, three to four mice to a pan, and were housed with animals receiving the same treatment. Animals were weighed on days 0 (day of surgery), 7, 10, and 14. Animals were all monitored for clinical signs of morbidity, including but not limited to paralysis and rapid wt. loss ( $>20 \%$ in 1 week). To determine statistical significance, a power analysis was performed using GPower3.1 software to verify the number of animals needed per experiment to achieve $80 \%$ power to detect a treatment effect of $30 \%$ compared with a baseline response of 5\% at a level of $\alpha=0.05$. After testing was completed, all mice were humanely sacrificed under deep ketamine/xylazine anesthesia by cutting the diaphragm to perform cardiac puncture for serum draw, followed by a rapid cervical dislocation.

Intramedullary Implantation of 66.1 Cells into Mouse Femur. The surgical procedure was performed as previously described (Sukhtankar et al., 2011; Lozano-Ondoua et al., 2013a). Mice were anesthetized with ketamine/xylazine $(9.0 \mathrm{mg} / \mathrm{ml}: 1.0 \mathrm{mg} / \mathrm{ml}$, i.p.). A lateral incision was performed to expose the thigh muscle. An arthrotomy was performed, and the condyles of the right distal femur were visualized. A hole was drilled in the intercondylar space to enter the medullary cavity. In total, $8 \times 10^{4} 66.1$ breast adenocarcinoma cells (P10-20) suspended in $5 \mu$ l of OPTI-MEM were injected into the intramedullary cavity with an injection cannula affixed via plastic tubing to a 10- $\mu$ l Hamilton syringe. Proper placement of the injector was affirmed through use of Faxitron X-ray imaging. After implantation of cells, the hole in the distal femur was sealed with bone cement. The arthrotomy was closed using 5-0 Vicryl suture, and the skin incision was closed using wound clips. Animals were allowed 7 days to recover prior to behavioral testing. Wound clips were removed on day 7.

Measurement of Spontaneous Pain Behaviors. Spontaneous pain (flinching and guarding) were measured both acutely and chronically. All behavioral testing for all treatment paradigms were performed at the same time of day, approximately within the 1st hour of the beginning of the light cycle (0800). Flinching is characterized by the rapid flexing of the ipsilateral hind paw while the animal was not moving and is counted by the number of flexing movements made. However, if the mouse shook its foot while walking, this was also counted as a flinch. Guarding is characterized by the animal holding the ipsilateral hind paw into a retracted position near or under the torso. All mice were baselined for these behaviors at day 0 prior to surgery. All animals that were included in the study were selected for measurements of spontaneous pain. Each animal was observed for 2 minutes at all time points, and both flinching and guarding were recorded (Lozano-Ondoua et al., 2010; Sukhtankar et al., 2011). For acute studies, the pain behaviors were measured at $0,30,60,90,120$, 180 , and 240 minutes after a single dose of MJN110 was administered into the intraperitoneal space. Measurements for the acute behaviors were measured from time $0-2,30-32,60-62,90-92,120-122$, 180-182, and 240-242 minutes after intraperitoneal injection of MJN110. For chronic studies, the animals were baselined at day 0 (before surgery) and tested at days 7, 10, and 14 after femoral cancer implants for spontaneous pain behaviors at the time point that corresponded to maximal response to drug from the acute study. The chronic administration of the various drugs were given at 
approximately the same time daily (0800) from days 7 to 14 postsurgery. Chronic pain behaviors were all measured 60 minutes after injection of the compound(s) at days 7, 10, and 14 postsurgery. Subsequent injections of test compounds were made on alternating sides to reduce damage to soft tissues. Antagonist studies were performed as previously described (Lozano-Ondoua et al., 2013a): animals were pretreated with the CB1 antagonist SR141716A $(1 \mathrm{mg} / \mathrm{kg})$ or the CB2 antagonist SR144528 $(1 \mathrm{mg} / \mathrm{kg}) 10$ minutes prior to the injection of MJN110 (10 mg/kg). All behaviors were performed and analyzed by a treatment-blinded observer throughout the course of the study who was designated to perform all behaviors to ensure consistency.

Radiographic Analysis. Mice were anesthetized using ketamine/ xylazine prior to obtaining radiographs using the digital Faxitron system. The images were obtained on days 0 (prior to surgery), 7, 10, and 14 and evaluated using a five-point bone rating scale by independent and blinded observers; scores were averaged. The bone scoring scale was as follows: $0=$ normal bone, $1=1-3$ lesions with no fracture, $2=4+$ lesions with no fracture, $3=$ unicortical, full-thickness fracture, and $4=$ bicortical, full-thickness fracture. Animals with full cortical fracture before day 14 were euthanized, and their data were excluded from analysis. Eight to 12 animals per group were analyzed for radiographic evidence of bone lesions to determine an average bone score.

\section{In Vitro and Ex Vivo}

Cell Culture. Murine 66.1 breast adenocarcinoma cells were obtained from Dr. Amy M. Fuller (Walser et al., 2006) and were maintained in Dulbucco's Modified Eagle Medium containing $10 \%$ FBS and 1\% penicillin-streptomycin. Cells were passaged every 4-5 days, harvested, and used for experiments or surgical implantation between passages 10 and 20 . Cells were incubated at $37^{\circ} \mathrm{C}$ with $5 \% \mathrm{CO}_{2}$.

Cell Viability Assay. The 66.1 adenocarcinoma cells were plated in 96-well plates at a concentration of $1 \times 10^{4}$ cells per well in full serum media. After 24 hours, the cells were then treated with respective pharmacological treatments in serum-free media. The treatments used were as follows: serum-free medium alone, vehicle (0.1\% DMSO), KML29 (100 nM, $1 \mu \mathrm{M}, 10 \mu \mathrm{M})$, or MJN110 (100 nM, $1 \mu \mathrm{M}, 10 \mu \mathrm{M})$ for another 24 hours. Cell viability and sensitivity to various drug treatments were evaluated with the tetrazolium salt XTT cell proliferation assay according to the manufacturer's protocols (catalogue \#30-1011K; ATCC, Manassas, VA).

Histomorphogenic Analysis and Tumor Burden. Animals were anesthetized with ketamine/xylazine intraperitoneally and euthanized on postsurgical day 14, and femurs were harvested for histology. Animals were perfused transcardially using $0.1 \mathrm{M}$ phosphate buffer, followed by a $4 \%$ paraformaldehyde solution in $0.1 \mathrm{M}$ phosphate buffer. Femurs were harvested, postfixed in $4 \%$ paraformaldehyde overnight at $4^{\circ} \mathrm{C}$, and decalcified in $10 \%$ EDTA solution (RDO-Apex, Aurora, IL) for 2 weeks. The EDTA buffer was changed every 3 days. Femurs were embedded in paraffin and sliced into $5-\mu \mathrm{m}$ frontal sections. Slides were rehydrated in progressively more-dilute ethanol solutions and stained with H\&E. Images were captured under bright-field microscopy using a Nikon E800 at $4 \times$ magnification. Bone morphology and percent tumor burden between the epiphyseal plates in the intramedullary cavity were assessed using ImageJ.

Pharmacological Agents. Animals received MJN110, the monoacylglycerol lipase (MAGL) inhibitor that was designed, synthesized, and gifted to the Vanderah laboratory from the laboratory of Dr. Benjamin Cravatt at the Scripps Research Institute (Niphakis et al., 2013). The CB1-specific antagonist SR141716A and SR144528, respectively, were obtained from Tocris and were delivered i.p. at a dose of $1 \mathrm{mg} / \mathrm{kg}$. All i.p. injections were made at a volume of $10 \mathrm{ml} / \mathrm{kg}$. Acute studies employed one injection of MJN110 (1, 3, or $10 \mathrm{mg} / \mathrm{kg}$, i.p.) and/ or antagonists ( $1 \mathrm{mg} / \mathrm{kg}$, i.p.). Chronic studies consisted of once-daily i.p. injections of MJN110 (1, 3, or $10 \mathrm{mg} / \mathrm{kg})$, with and without antagonists ( $1 \mathrm{mg} / \mathrm{kg}$, i.p.), from days 7 to 14 postsurgery; the side of injection was alternated daily. MJN110 and antagonists were dissolved in a vehicle solution of $10 \%$ DMSO, $10 \%$ Tween-80, and $80 \%$ normal saline for all animal studies. Control animals received vehicle solution alone after the same dosing regimen.

Statistical Analysis. All data were analyzed using either one-way or ordinary and repeated measure two-way ANOVA depending on experimental protocol with Bonferroni post hoc corrections. Power analysis was performed on cumulated data using GPower3.1 software, and we found the adequate statistical separation for each group to detect 0.80 between groups at $P<0.05$. Data were expressed as means \pm S.D. or S.E.M., with data analysis performed using GraphPad Prism 7.0 (Graph Pad Inc., San Diego, CA). All studies were performed in a blinded-to-treatment manner.

\section{Results}

MJN110 is Effective at Relieving Acute Pain Behaviors in a Murine Model of Metastatic Breast Cancer. To study the effects of MJN110 on bone pain derived from metastatic breast cancer, we used an established murine model that reliably replicates pain in long bones due to cancer (Lozano-Ondoua et al., 2013a). In this syngeneic model, the 66.1 breast adenocarcinoma cell line was introduced into the medullary canal of healthy female BALB/c mice and was allowed to seed the site for 7 days. On day 7, flinching and guarding behaviors were observed and recorded to obtain a postcancer baseline (CIBP). All animals were injected with either vehicle or 1-, 3-, or 10-mg/kg of MJN110, and behaviors were recorded at $30,60,90,120,180$, and 240 minutes after injection to construct a dose response curve. Compared withvehicle control, the MJN110 dose dependently decreased the observed spontaneous flinching behaviors. Significant decreases from time-matched vehicle controls were achieved by the $10-\mathrm{mg} / \mathrm{kg}$ dose at the 30-, 60-, 90-, and 240-minute time points (Fig. 1A). There was no significant difference at any time point or dose after MJN110 administration for the guarding behaviors (Fig. 1B). The time point at which maximal effect was observed for flinching was 60 minutes; hence, this time point was used for the chronic studies.

A dose response curve was generated at the time of maximal effect for flinching (60 minutes). The percent antinociception was calculated for each dose, and the $\mathrm{EC}_{50}$ was determined to be $2.093 \mathrm{mg} / \mathrm{kg}$ (95\% Confidence Interval $1.246-3.34 \mathrm{mg} / \mathrm{kg}$ ) for flinching behavior (Fig. 1D).

Chronic MJN110 Dosing Causes Significant Reduction in Pain Behaviors. After the acute studies, the groups were dosed once daily in the morning with vehicle or MJN110 (1, 3, or $10 \mathrm{mg} / \mathrm{kg}$ ) from days 7 to 14 postsurgery to study the effects of chronic treatment on pain and disease progression. Behavior was measured on days 10 and 14 postsurgery at baseline and up to 90 minutes postinjection to monitor the efficacy of MJN110 over time. As in the acute study, on day 10 postsurgery, the injections dose dependently decreased flinching behaviors 60 minutes after injection. Compared with time-matched vehicle control and CIBP baseline, all three doses significantly lowered the flinching behaviors observed $(2.69,2.11$, and 1.93 flinches vs. 5.75; $P<0.01$, Fig. 2 A). The maximal effect was still observed at 60 minutes postinjection, as was observed in the acute studies. As in the acute study, no significant differences in guarding behaviors were noticed across all the groups and time points (Fig. 2B). 

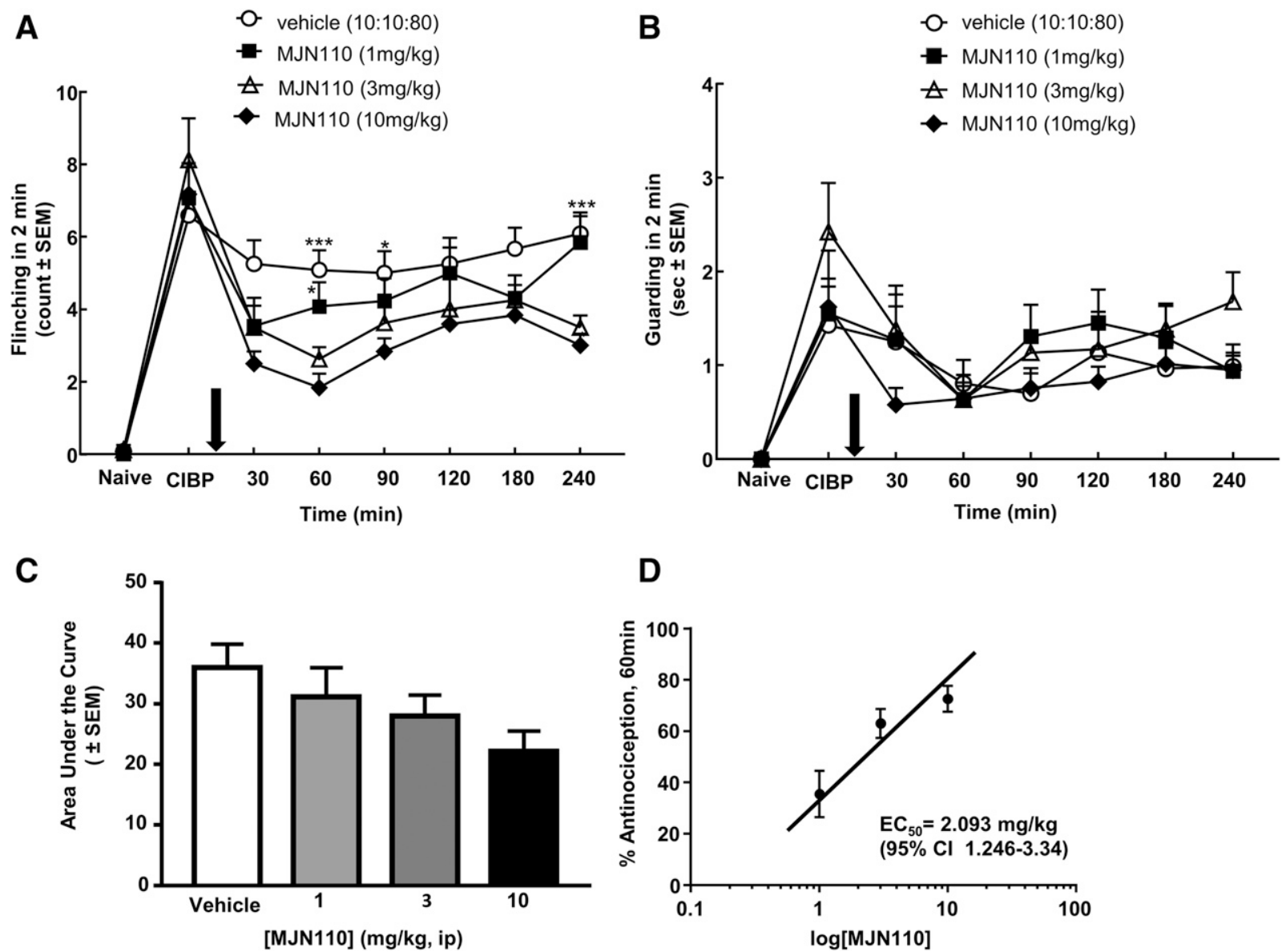

Fig. 1. MAGL inhibitor MJN110 acutely reverses established spontaneous pain induced by CIBP. (A) Flinching was dose dependently and significantly reduced by systemic injection of MJN110 (i.p.) 7 days after femoral inoculation with 66.1 breast adenocarcinoma cells. (B) Guarding induced by CIBP was significantly attenuated with the highest dose of MJN110 evaluated on D7 post-CIBP induction. Arrows indicate beginning of daily drug injections. (C) Area under the curve was determined from the acute flinching time course for each dose tested. (D) Dose response curve was generated, and EC $\mathrm{E}_{50}$ was determined to be $2.093 \mathrm{mg} / \mathrm{kg}$ (95\% CI 1.246-3.34). *P $<0.05 ; * * P<0.01$, ***P $<0.001 \mathrm{n}=8-12$ animals per group (two-way RM ANOVA, Bonferroni). CI, confidence interval; D, day; RM, repeated measures; CB1R, cannabinoind receptor 1; BL, baseline.

On day 14 postsurgery, flinching and guarding behaviors were observed again at 60 minutes postinjection. After the trend from both acute and day- 10 studies, treatment with MJN110 at 3 and $10 \mathrm{mg} / \mathrm{kg}$ significantly decreased the flinching behaviors observed compared with vehicle control (1.89 and 2.69 flinches vs. 6.25 flinches, respectively; $P<0.01$ ), but there was no significant difference with the $1-\mathrm{mg} / \mathrm{kg}$ dose (Fig. 2A). The guarding behaviors were not significantly different from vehicle at any dose tested (Fig. 2B). Moving forward, only flinching behavior was assessed and reported for the antagonist studies.

CB1 and CB2 Receptors Mediate MJN110 Effects on Spontaneous Pain Behaviors. To elucidate the receptor dependence of the effects in vivo, we used SR141716A and SR144528 to selectively inhibit CB1 and CB2 activation, respectively. Previously published work from our laboratory has demonstrated that these antagonists alone do not elicit effects for the outcome measures that we are reporting (Lozano-Ondoua et al., 2013a). All behavioral tests for the antagonists were performed at 60 minutes postinjection, the time point at which maximal effect was seen for MJN110. On day 7 post-CIBP surgery, the pharmacological inhibition of either CB1 or CB2 receptors blocked the analgesic effects of MJN110 at the $10-\mathrm{mg} / \mathrm{kg}$ dose (flinch count $=4.78$ and $5.40 \mathrm{vs}$.
1.79; $P<0.01)$. This same finding was seen with chronic dosing at days 10 and 14 . On day 14, blockade with CB1 antagonist SR141716A followed by MJN110 administration caused flinching behavior to increase significantly higher than vehicle and/or the CB2/MJN110 groups (Fig. 3, 11.00 vs. 6.46 and 5.90, respectively; $P<0.01$ ).

Chronic MJN110 has No Effect on Bone Disease as Observed on Radiographic Analysis. To assess bone morphology and disease progression, radiographs were obtained via Faxitron throughout the duration of the study. Presurgery, there was no difference between the groups $(P>0.999)$. At day 14 postsurgery, there was no significant difference between any of the groups tested, therefore suggesting MJN110 has no effect on bone disease progression as determined by radiographic evaluation (Fig. 4).

66.1 Cell Viability is Decreased in Response to MJN110 In Vitro. To assess the effects of MJN110 on cancer cell viability, an XTT assay was performed in the presence of varying concentrations of MJN110. MJN110 was able to significantly decrease the cell viability of 66.1 cells in a dose-dependent manner at doses of $100 \mathrm{nM}(P<0.05)$, $1 \mu \mathrm{M}(P<0.05)$, and $10 \mu \mathrm{M}(P<0.0001)$ when compared with $0.1 \%$ DMSO as vehicle control (Fig. 5A). These experiments were then repeated with an alternate MAGL inhibitor 

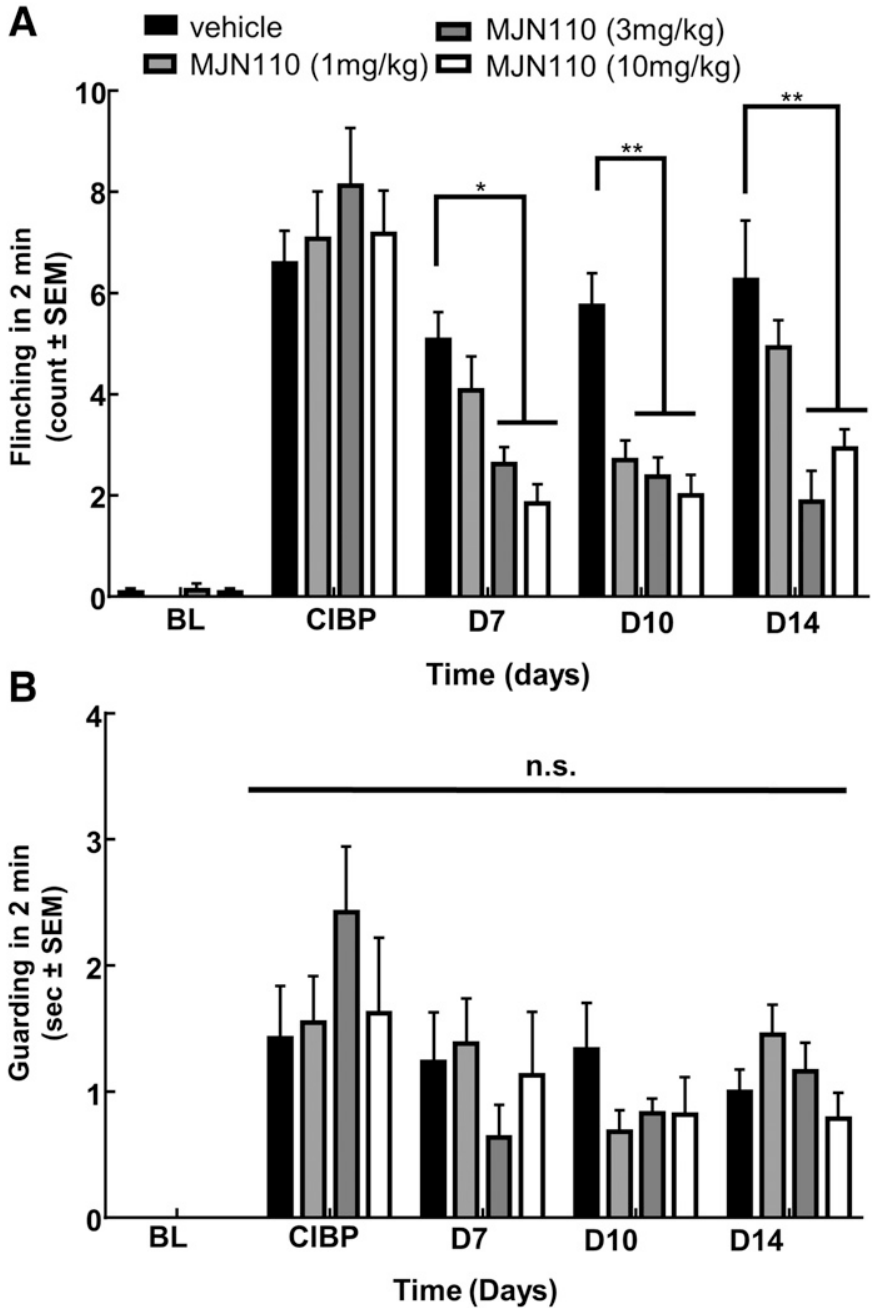

Fig. 2. MAGL inhibitor MJN110 dosed chronically reverses established flinching induced by CIBP. (A) Flinching was dose dependently and significantly reduced by systemic injection of MJN110 (once daily, 7 days) after femoral inoculation with 66.1 breast adenocarcinoma cells. (B) Guarding behaviors were not significantly reduced with chronic dosing of MJN110. Daily drug injections began immediately following CIBP baseline measurements. ${ }^{*} P<0.05$; ${ }^{* *} P<0.01, n=8-12$ animals per group (Two-way RM ANOVA, Bonferroni test). BL, baseline; D, day.

(KML29) and a CB2 agonist (JWH015). Each drug modestly decreased the viability of 66.1 cells compared with MJN110 over the same concentrations, although significance was only seen at the highest dose (data not shown). A dose response curve was generated for the three compounds, and MJN110 was shown to be more potent and efficacious at decreasing 66.1 cell viability in vitro over the tested concentrations (Fig. 5B).

66.1 Cell Proliferation is Decreased in Response to MJN110 in Vitro. To better ascertain whether the decrease in viability seen in the XTT assay was due to changes in proliferation or due to direct cytotoxicity of the drug, we performed a 5-bromo-2'-deoxyuridine (BrdU) assay on 66.1 cells in varying concentrations of MJN110. MJN110 was able to significantly decrease the proliferation of 66.1 cells at the highest dose tested ( $10 \mu \mathrm{M}, P<0.0001$, Fig. $5 \mathrm{C})$. Similar to the XTT assay, we repeated the experiment with an alternative and chemically distinct MAGL inhibitor, KML29, and the CB2 agonist JWH015. KML29 had no effect at any tested dose on proliferation of the 66.1 cells (data not shown), whereas
JWH015 had a similar effect at decreasing proliferation as MJN110 at the highest dose, but not to the same degree (Fig. 6A). A dose response curve was generated for the compounds, and it was demonstrated that MJN110 and JWH015 decreased cellular proliferation (Fig. 6B).

Tumor Burden in Vivo is Not Reduced by Chronic MJN110 Treatment. On day 14, mice were sacrificed, and femurs were collected for histology to assess how the treatments affected the tumor burden within the bone. Bones were harvested, fixed, and decalcified prior to paraffin embedding and sectioning prior to $\mathrm{H} \& \mathrm{E}$ staining. Using ImageJ software (National Institutes of Health), the tumor burden was quantified by measuring the tumor area within the medullary canal between the epiphyseal plates. Bones were collected from the vehicle and $10-\mathrm{mg} / \mathrm{kg}$ animals and analyzed for tumor burden to capture the effects of MJN110 at the highest tested dose. Chronic dosing of MJN110 at $10 \mathrm{mg} / \mathrm{kg}$ in animals did not produce a significant change in the tumor burden measured within the sections compared with vehicle control (Fig. 7, $P=0.47$ )

\section{Discussion}

Because limited therapies are available for metastatic bone cancer pain, identification and validation of potentially disease modifying therapeutics is necessitated. Studies above evaluated whether increasing endogenous cannabinoid tone by inhibiting MAGL would attenuate nocifensive behaviors in a murine model of metastatic breast cancer. Here, we report that the MAGL inhibitor MJN110 dose dependently decreased cancer-induced, spontaneous pain

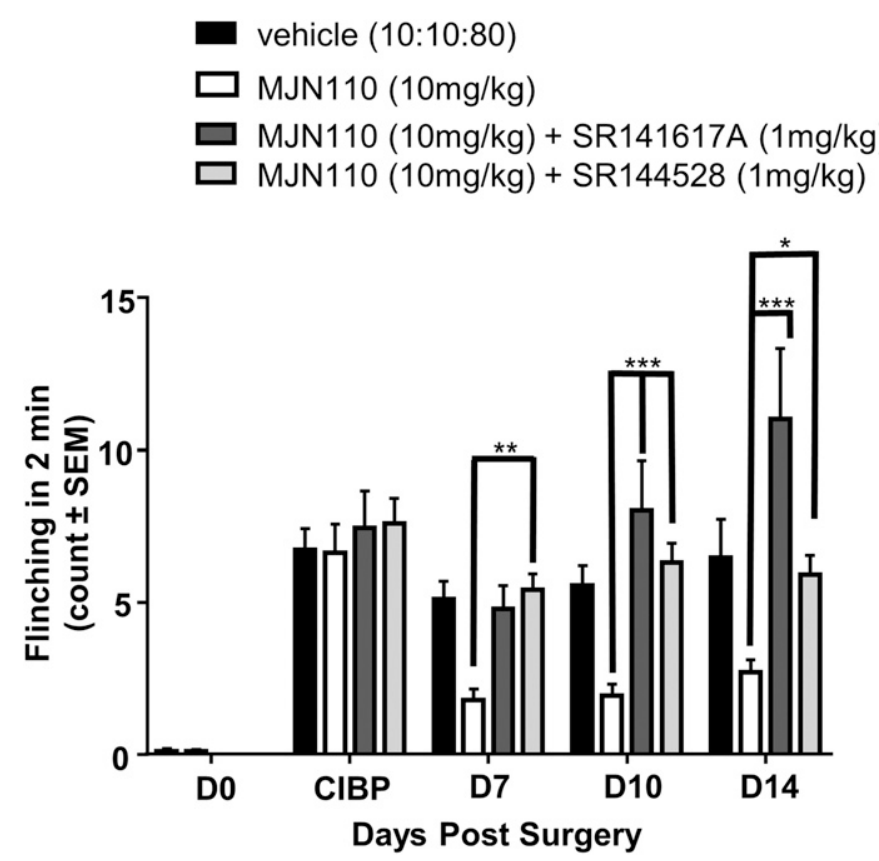

Fig. 3. Both CB1 and CB2 antagonists attenuate MJN110 effects against cancer-induced flinching behaviors. Both coadministration of the CB1R antagonist SR141716A (1 mg/kg) with MJN110 or the CB2R antagonist SR144528 (1 mg/kg) significantly reversed MJN110 attenuation of flinching induced by CIBP.*P $<0.05 ; * * P<0.01 ; * * * P<0.001, n=$ 8-12 animals per group (two-way RM ANOVA, Bonferroni test). CB1R, $\mathrm{CB} 1$ receptor; $\mathrm{D}$, day; $\mathrm{RM}$, repeated measures. 
A
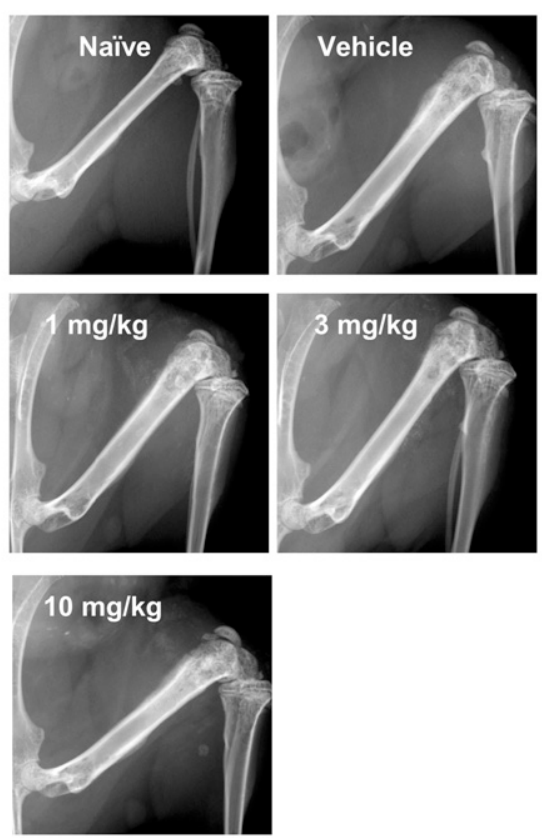

B

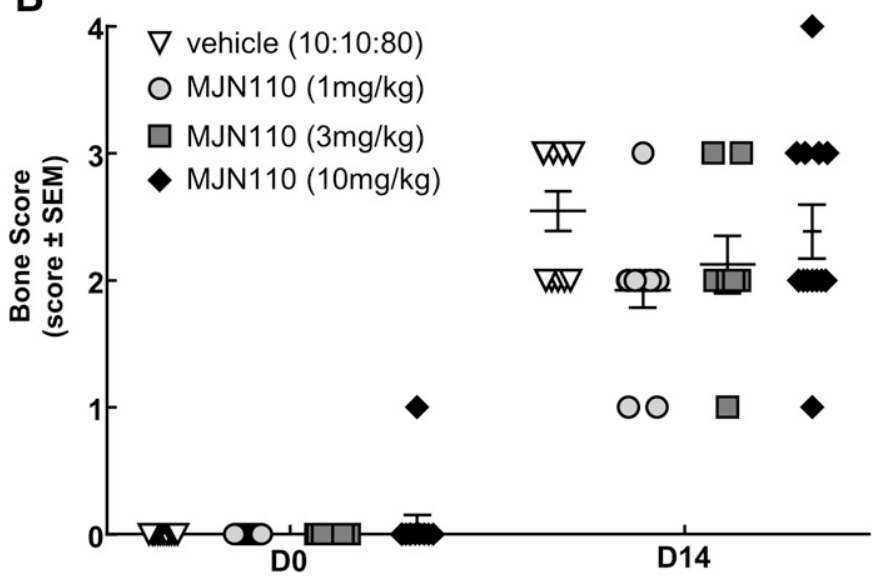

Fig. 4. MJN110 had no effect on radiographic evidence of cancer-induced bone lesions. (A) Radiographs were taken of the ipsilateral femur at D0 prior to surgery and at D14 at the end of the study. Compared with vehicle control, there was no significant difference found between groups $(\mathrm{F}=$ 1.205, $P=0.4704)$. Images displayed are representative at D14 postsurgery. (B) Bones were evaluated using a five-point bone rating scale by three blinded individuals, and scores were averaged. The bone scoring scale was as follows: $0=$ normal bone, $1=1-3$ lesions with no fracture, $2=4+$ lesions with no fracture, $3=$ unicortical, full-thickness fracture, and 4 = bicortical, full-thickness fracture (two-way RM ANOVA, Bonferroni test). D, day; RM, repeated measures.

behaviors in mice, suggesting that targeting the endocannabinoid system may be a novel strategy to mitigate CIBP.

MJN110, a prototypical MAGL inhibitor, has shown to be effective at alleviating neuropathic pain in animal models (Burston et al., 2016; Wilkerson et al., 2016). CIBP displays characteristics of neuropathic and inflammatory pain (LozanoOndoua et al., 2013b; Slosky et al., 2016); thus, targeting pain of inflammatory and neuropathic origin is rational. MAGL inhibition increases 2-AG levels to inhibit nociceptive fibers via activation of $\mathrm{CB} 1 / \mathrm{CB} 2$ receptors and reduces levels of arachidonic acid, the precursor for pronociceptive/inflammatory prostaglandins (Nomura et al., 2011).
Cancer patients often experience pain at rest or while ambulating, suggesting that spontaneous or movementevoked pain behaviors more accurately reflect types of daily pain a patient would experience compared with mechanically evoked measures, such as von Frey (Delaney et al., 2008; Currie et al., 2013). Our hypothesis was that in acute and chronic dosing, systemic administration of MJN110 would significantly attenuate pain behaviors of flinching or guarding as compared with vehicle; typically, animals will display either flinching (spontaneous pain), guarding (ongoing pain), or mixed behaviors. Although flinching and guarding often are grouped together as "spontaneous" behaviors, our studies demonstrate that these may be distinct. Our data demonstrate that a single injection of MJN110 decreased flinching behaviors compared with vehicle up to 90 minutes after systemic administration at $10 \mathrm{mg} / \mathrm{kg}$. This is consistent with the literature, as studies demonstrating antiallodynia and antinociceptive effects of MAGL inhibition only evaluate behavior in the chronic setting or at least 2 hours after systemic administration (Ghosh et al., 2013; Burston et al., 2016; Wilkerson et al., 2016). Interestingly, four hours postinjection, MJN110 at 3 and $10 \mathrm{mg} / \mathrm{kg}$ emerged as significantly different from vehicle, suggesting either an active metabolite or latent effects of the parent compound due to compartmental release. Given that MJN110 inhibits MAGL up to $70 \%$ after a single dose and that this inhibition is maintained for 12 hours (Niphakis et al., 2013), the resurgence of antinocifensive activity at higher doses may represent a novel pharmacokinetic interaction with other molecular systems.
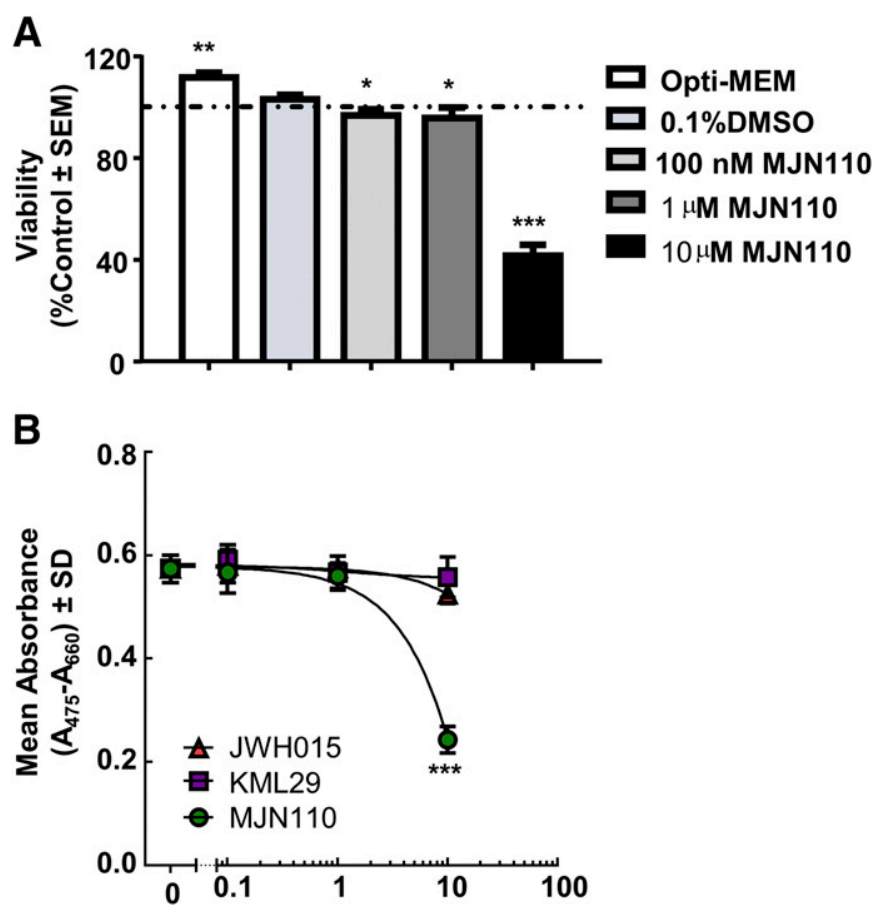

[Drug], $\mu \mathrm{M}$

Fig. 5. Effects of MJN110 on 66.1 cancer cell viability in vitro. (A) Across three concentrations tested, MJN110 significantly decreased the cell viability of 66.1 cells in vitro at the $10-\mu \mathrm{M}$ dose as assessed by XTT assay. One-way ANOVA with Bonferroni post hoc correction. ${ }^{*} P<0.05$; $* * P<$ 0.01 ; $* * * P<0.001(n=8)$. (B) Dose response curve was generated for MJN110 and compared with the CB2 agonist JWH015 and the MAGL inhibitor KML29 to determine effects on cell viability of 66.1 cells in vitro. 


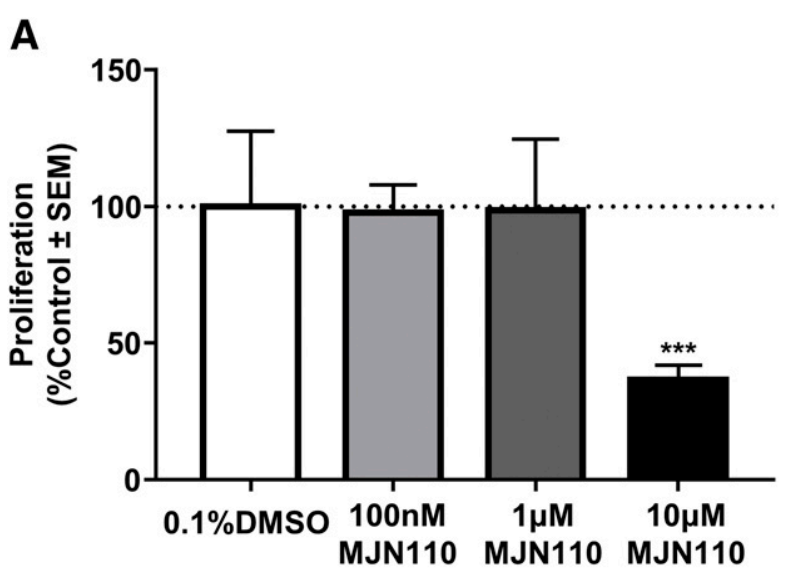

B

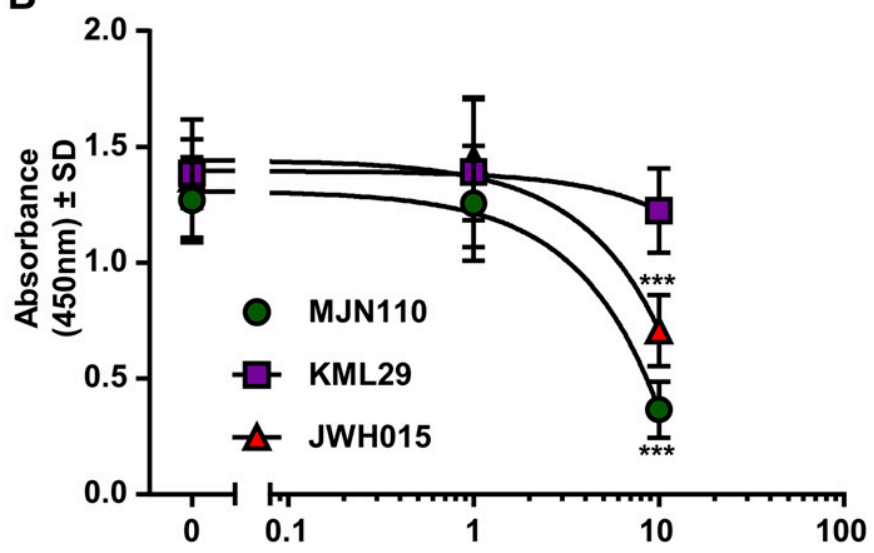

Fig. 6. Effects of MJN110 on 66.1 cancer cell proliferation in vitro. BrdU assay was performed to better assess changes in proliferation of 66.1 cells in response to MJN110. (A) Across three concentrations tested, MJN110 significantly decreased the proliferation of 66.1 cells in vitro at the $10-\mu \mathrm{M}$ dose. One-way ANOVA with Bonferroni post hoc correction. ${ }^{* * * P}<0.001$ ( $n=8$ animals per group). (B) Dose response curve was generated for MJN110 and compared with the CB2 agonist JWH015 and the alternative MAGL inhibitor KML29 to determine effects on cell proliferation in vitro. BrdU = 5-bromo-2'-deoxyuridine.

The efficacy of MJN110 after chronic administration increased over time. This is in contrast to reports in which high doses of MJN110 leading to $\mathrm{CB}$ receptor desensitization caused the drug to lose efficacy during chronic treatment, but it is consistent with studies demonstrating that lower doses maintain efficacy with persistent treatment (Schlosburg et al., 2010; Burston et al., 2016). Different from the acute study, all three doses demonstrated significant differences from time-matched vehicle, whereas acutely, only $10 \mathrm{mg} / \mathrm{kg}$ showed a significant difference at 60 minutes. These results demonstrate that chronic dosing regimens promote sensitization to the drug during CIBP to elicit a more profound effect at lower doses. Our behavioral data suggest that in CIBP, CB receptors may not lose their sensitivity to [2-AG] when MAGL is inhibited. Additionally, in metastatic cancer, there is ongoing proliferation and increases in inflammatory factors and tissue damage (David Roodman, 2003; Landskron et al., 2014) that may be reduced not only by CB receptor activation but also by reduction in production of prostaglandins by chronic MAGL inhibition.

It is suggested that MAGL inhibitors elicit their acute analgesic effects through the CB1 receptors (Long et al., 2009 ), yet in reports on neuropathic and inflammatory pain models, the CB2 receptor has a more prominent role in antihyperalgesic effects (Manzanares et al., 2006). Since CIBP displays aspects of inflammatory and neuropathic pain, it is reasonable that both $\mathrm{CB} 1$ and $\mathrm{CB} 2$ receptors may be viable targets for inducing analgesia. After neuropathic injury, CB2 receptor expression increases in the spinal dorsal horn and sensory neurons, which makes this a viable target (Beltramo et al., 2006). Mice pretreated with CB1 antagonist SR141716A or CB2 antagonist SR144528 demonstrated significant attenuation in MJN110 antihyperalgesia, suggesting that both $\mathrm{CB} 1$ and $\mathrm{CB} 2$ receptors are contributing. As previously reported, these antagonists have
A

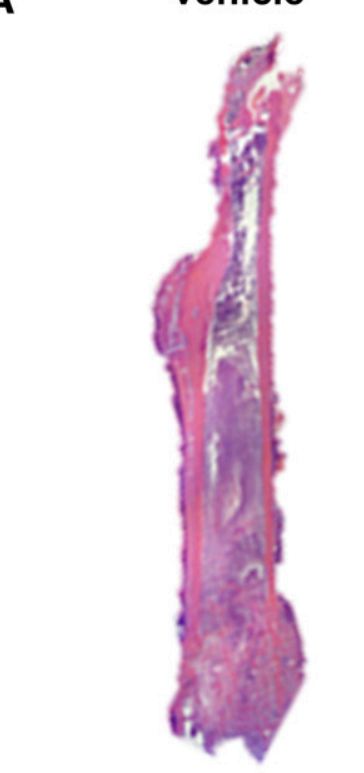

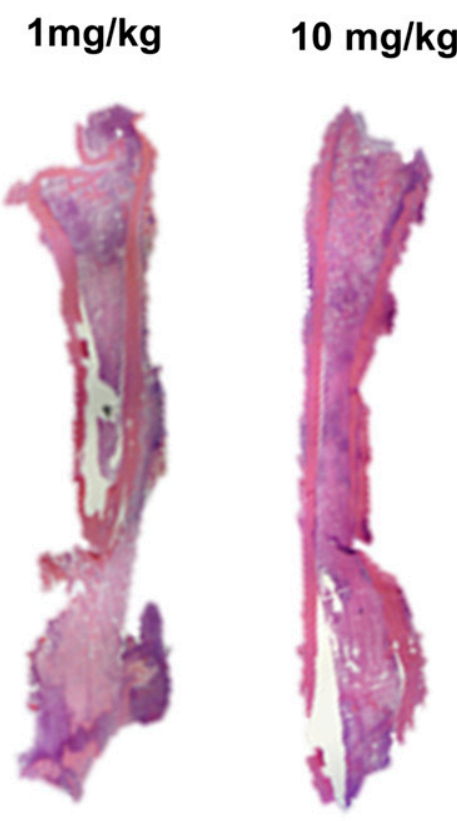

B

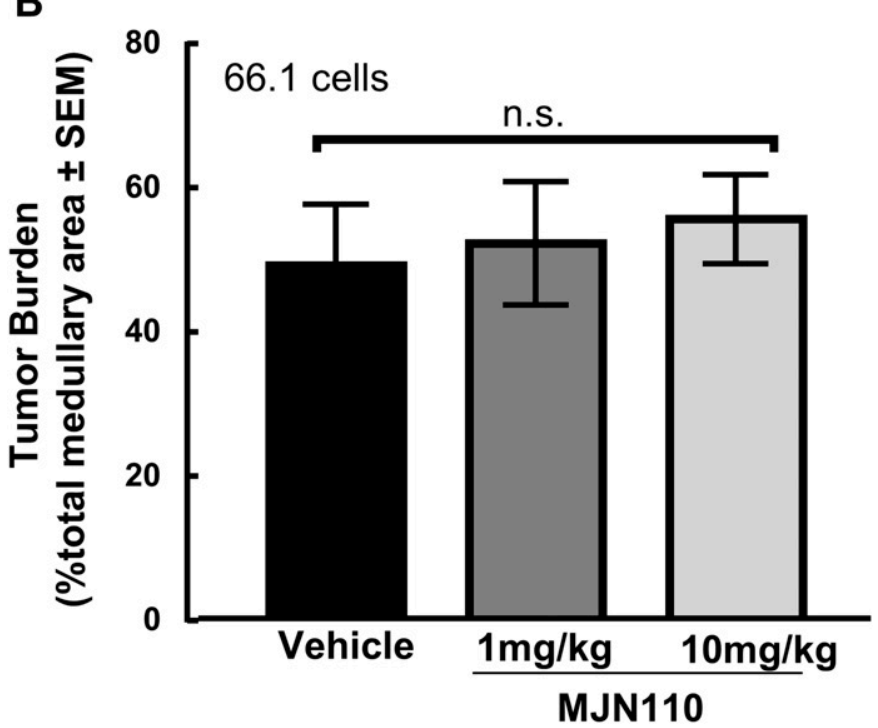

Fig. 7. Effects of MJN110 on 66.1 cancer cells in vivo. (A) Representative H\&E images of cross sections of femurs from animals treated with vehicle or doses of 1 and $10 \mathrm{mg} / \mathrm{kg}$ of MJN110 once a day for 7 days. Imaged at original magnification, $4 \times$. (B) H\&E images were then quantified for tumor burden, which demonstrated that there was no significant change in tumor burden within the medullary cavity across the doses tested $(\mathrm{F}=$ $0.1956, P=0.8297$ ). One-way ANOVA with Tukey's multiple comparisons post hoc test ( $n=3$ animals per group). n.s $=$ no significance. 
no effect on spontaneous pain behaviors alone in this model (Lozano-Ondoua et al., 2013a), suggesting that 2-AG levels may be low because of the lack of production or the enhanced breakdown of 2-AG by MAGL, which has been shown to increase after injury (Wilkerson et al., 2012), resulting in increased production of AA, which may lead to increased prostaglandins and inflammation.

Previous studies demonstrated that exogenous agonism of the CB1/CB2 receptors improve bone health in CIBP models (Lozano-Ondoua et al., 2010, 2013a). After 7 days of MJN110 intervention, a modest but insignificant decrease in the bone scores for the $1-\mathrm{mg} / \mathrm{kg}$ but not the 3 - and $10-\mathrm{mg} / \mathrm{kg}$ groups suggest that lower doses of MJN110 may preserve bone. It remains unknown why higher doses are not demonstrating a similar trend. One possibility is the loss of arachidonic acid and prostaglandin production that results from MAGL inhibition. Although AA and prostaglandins are known to promote inflammatory pain, they are necessary for maintaining the integrity of bone. Orthopedic literature indicates that chronic use of NSAIDs, which inhibit cyclooxygenase enzymes, deters bone healing (Ho et al., 1999; Krischak et al., 2007; Giannoudis et al., 2015). Prostaglandins play a role in healing by modulating osteoblasts and osteoclasts. Prostaglandin E2 was shown to be a potent stimulator of bone resorption by enhancing osteoclast formation via indirect action through stromal cells and by acting at osteoclast prostaglandin E2 receptor 4 (EP4) receptors directly (Brändström et al., 1998). Other studies demonstrated that prostaglandin F2 and prostaglandin E2 stimulate osteoblast proliferation and differentiation (Hakeda et al., 1987). Quantitative, unbiased measurements of different prostaglandins in the bone-tumor microenvironment as well as prostaglandin receptor type and location need to be further explored.

Cannabinoid receptor agonism reduces tumor burden in preclinical CIBP models (Lozano-Ondoua et al., 2010, 2013a,b). Moreover, it was demonstrated that MAGL overexpression increased the metastatic potential of normally nonaggressive tumors (Nomura et al., 2010). After repeated MJN110 exposure, we examined tumor burden by measuring the percentage of tumor occupying the medullary cavity. We did not find a significant difference between vehicle and the highest dose tested, nor did we observe any distant metastasis in either group. Literature suggests that MAGL is highly expressed in aggressive, metastatic tumors, including breast cancers, and is a marker for invasiveness in various tumor cell lines (Nomura et al., 2010; Baba et al., 2017). Our minimal observed effects of MJN110 on tumor growth and burden warrant further investigation at multiple doses before ruling out whether MJN110 may alter tumor burden in this model. To further evaluate the effects of MJN110 on tumor cell viability and proliferation, we examined the effects of two MAGL inhibitors on 66.1 cell viability. We demonstrated that JWH015, KML29, and MJN110 can decrease in vitro viability, but MJN110 was more potent than the other MAGL inhibitor or a CB2 agonist alone. Additionally, we tested the effects of MJN110 on proliferation by 5-bromo-2'-deoxyuridine (BrdU) assay and noticed that MJN110 significantly decreased proliferation of 66.1 cells, which indicated that the decrease in cell viability was likely due to decreased cellular proliferation rather than cytotoxicity. This increased observed potency is likely due to the differential chemistry between KML29 and MJN110 and how they bind and inactivate serine hydrolases
(Niphakis et al., 2013). The discrepancy between the in vitro studies and the in vivo tumor burden was surprising. We hypothesize that it could be due to differences in pharmacokinetics when administered in vivo that lead to a subthreshold dose at the tumor site to elicit the antiproliferative effects; higher doses may be tried to elicit the antitumor effects. Other anticancer therapeutics that demonstrate strong in vitro antitumor abilities can have minimal effects in vivo depending on dose and routes of administration (Theiner et al., 2015). Therefore, pharmacokinetics and administration route should be considered, and higher doses may elicit antitumor effects, although these have not been studied to date.

In this first report using MJN110 in a mouse CIBP model, results strongly suggest that MAGL inhibition is effective at reversing pain behaviors by indirectly activating CB1 and CB2 receptors. The novel finding that MJN110 works acutely (within 30 minutes) and chronically (after a few hours to days) introduces an option that is effective against breakthrough and ongoing pain while lacking analgesic tolerance. Additionally, we have shown that MJN110 may increase potency when administered chronically while maintaining efficacy at high doses. This is promising for the fact that, compared with other MAGL inhibitors, MJN110 elicits antinociception without demonstrating cannabimimetic effects like hypothermia, catalepsy, or hypomotility (Ignatowska-Jankowska et al., 2015). Additionally, we demonstrate that, at lower doses, MJN110 may be beneficial in the preservation of bone health despite cancer progression and that chronic treatment of MJN110 does not enhance tumor burden in vivo and may slow proliferation in vitro. Together, our data suggest that inhibiting MAGL to increase endogenous cannabinoid tone may be a viable mechanism with clinical application to treat chronic metastatic cancer-induced pain.

\section{Authorship Contributions}

Participated in research design: Thompson, Grenald, LargentMilnes, Vanderah.

Conducted experiments: Thompson, Grenald, Ciccone, BassiriRad. Contributed new reagents or analytic tools: Niphakis, Cravatt. Performed data analysis: Thompson, Largent-Milnes, Vanderah. Wrote or contributed to the writing of the manuscript: Thompson, Largent-Milnes, Vanderah.

\section{References}

Baba Y, Funakoshi T, Mori M, Emoto K, Masugi Y, Ekmekcioglu S, Amagai M, and Tanese $\mathrm{K}(2017)$ Expression of monoacylglycerol lipase as a marker of tumour invasion and progression in malignant melanoma. $J$ Eur Acad Dermatol Venereol 31:2038-2045.

Beltramo M, Bernardini N, Bertorelli R, Campanella M, Nicolussi E, Fredduzzi S, and Reggiani A(2006) CB2 receptor-mediated antihyperalgesia: possible direct involvement of neural mechanisms. Eur J Neurosci 23:1530-1538.

Brändström H, Jonsson KB, Ohlsson C, Vidal O, Ljunghall S, and Ljunggren O(1998) Regulation of osteoprotegerin mRNA levels by prostaglandin E2 in human bone marrow stroma cells. Biochem Biophys Res Commun 247:338-341.

Burston JJ, Mapp PI, Sarmad S, Barrett DA, Niphakis MJ, Cravatt BF, Walsh DA, and Chapman V(2016) Robust anti-nociceptive effects of monoacylglycerol lipase inhibition in a model of osteoarthritis pain. Br J Pharmacol 173:3134-3144.

Coleman RE(2006) Clinical features of metastatic bone disease and risk of skeletal morbidity. Clin Cancer Res 12:6243s-6249s.

Currie GL, Delaney A, Bennett MI, Dickenson AH, Egan KJ, Vesterinen HM, Sena ES, Macleod MR, Colvin LA, and Fallon MT(2013) Animal models of bone cancer pain: systematic review and meta-analyses. Pain 154:917-926.

Curry ZA, Wilkerson JL, Bagdas D, Kyte SL, Patel N, Donvito G, Mustafa MA, Poklis JL, Niphakis MJ, Hsu KL, et al.(2018) Monoacylglycerol lipase inhibitors reverse paclitaxel-induced nociceptive behavior and proinflammatory markers in a mouse model of chemotherapy-induced neuropathy. J Pharmacol Exp Ther 366:169-183. David Roodman G(2003) Role of stromal-derived cytokines and growth factors in bone metastasis. Cancer 97 (3 Suppl):733-738.

Delaney A, Fleetwood-Walker SM, Colvin LA, and Fallon M(2008) Translational medicine: cancer pain mechanisms and management. Br J Anaesth 101:87-94. 
Dinh TP, Carpenter D, Leslie FM, Freund TF, Katona I, Sensi SL, Kathuria S, and Piomelli $\mathrm{D}(2002)$ Brain monoglyceride lipase participating in endocannabinoid inactivation. Proc Natl Acad Sci USA 99:10819-10824.

Dinh TP, Kathuria S, and Piomelli D(2004) RNA interference suggests a primary role for monoacylglycerol lipase in the degradation of the endocannabinoid 2arachidonoylglycerol. Mol Pharmacol 66:1260-1264.

Ghosh S, Wise LE, Chen Y, Gujjar R, Mahadevan A, Cravatt BF, and Lichtman AH(2013) The monoacylglycerol lipase inhibitor JZL184 suppresses inflammatory pain in the mouse carrageenan model. Life Sci 92:498-505.

Giannoudis PV, Hak D, Sanders D, Donohoe E, Tosounidis T, and Bahney C(2015) Inflammation, bone healing, and anti-inflammatory drugs: an update. J Orthop Trauma 29 (Suppl 12):S6-S9.

Hakeda Y, Hotta T, Kurihara N, Ikeda E, Maeda N, Yagyu Y, and Kumegawa M(1987) Prostaglandin E1 and F2 alpha stimulate differentiation and proliferation, respectively, of clonal osteoblastic MC3T3-E1 cells by different second messengers in vitro. Endocrinology 121:1966-1974.

Hanlon KE, Lozano-Ondoua AN, Umaretiya PJ, Symons-Liguori AM, Chandramouli A, Moy JK, Kwass WK, Mantyh PW, Nelson MA, and Vanderah TW(2016) Modulation of breast cancer cell viability by a cannabinoid receptor 2 agonist, JWH015, is calcium dependent. Breast Cancer (Dove Med Press) 8:59-71.

Ho ML, Chang JK, Chuang LY, Hsu HK, and Wang GJ(1999) Effects of nonsteroidal anti-inflammatory drugs and prostaglandins on osteoblastic functions. Biochem Pharmacol 58:983-990.

Hohmann AG, Suplita RL, Bolton NM, Neely MH, Fegley D, Mangieri R, Krey JF, Walker JM, Holmes PV, Crystal JD, et al.(2005) An endocannabinoid mechanism for stress-induced analgesia. Nature 435:1108-1112.

Ibrahim MM, Porreca F, Lai J, Albrecht PJ, Rice FL, Khodorova A, Davar G, Makriyannis A, Vanderah TW, Mata HP, et al.(2005) CB2 cannabinoid receptor activation produces antinociception by stimulating peripheral release of endogenous opioids. Proc Natl Acad Sci USA 102:3093-3098.

Ibrahim MM, Rude ML, Stagg NJ, Mata HP, Lai J, Vanderah TW, Porreca F, Buckley NE, Makriyannis A, and Malan TP Jr.(2006) CB2 cannabinoid receptor mediation of antinociception. Pain 122:36-42.

Ignatowska-Jankowska B, Wilkerson JL, Mustafa M, Abdullah R, Niphakis M, Wiley JL, Cravatt BF, and Lichtman $\mathrm{AH}(2015)$ Selective monoacylglycerol lipase inhibitors: antinociceptive versus cannabimimetic effects in mice. J Pharmacol Exp Ther 353:424-432

Jimenez-Andrade JM, Mantyh WG, Bloom AP, Ferng AS, Geffre CP, and Mantyh PW(2010) Bone cancer pain. Ann N Y Acad Sci 1198:173-181.

Jin J(2015) JAMA PATIENT PAGE. Nonsteroidal anti-inflammatory drugs. JAMA 314:1084.

King T, Vardanyan A, Majuta L, Melemedjian O, Nagle R, Cress AE, Vanderah TW, Lai J, and Porreca F(2007) Morphine treatment accelerates sarcoma-induced bone pain, bone loss, and spontaneous fracture in a murine model of bone cancer. Pain 132:154-168.

Kohnz RA and Nomura DK(2014) Chemical approaches to therapeutically target the metabolism and signaling of the endocannabinoid 2-AG and eicosanoids. Chem Soc Rev 43:6859-6869.

Krischak GD, Augat P, Sorg T, Blakytny R, Kinzl L, Claes L, and Beck A(2007) Effects of diclofenac on periosteal callus maturation in osteotomy healing in an animal model. Arch Orthop Trauma Surg 127:3-9.

Landskron G, De la Fuente M, Thuwajit P, Thuwajit C, and Hermoso MA(2014) Chronic inflammation and cytokines in the tumor microenvironment. $J$ Immunol Res 2014:149185.

Long JZ, Li W, Booker L, Burston JJ, Kinsey SG, Schlosburg JE, Pavón FJ, Serrano AM, Selley DE, Parsons LH, et al.(2009) Selective blockade of 2 arachidonoylglycerol hydrolysis produces cannabinoid behavioral effects. Nat Chem Biol 5:37-44.

Lozano-Ondoua AN, Hanlon KE, Symons-Liguori AM, Largent-Milnes TM, Havelin JJ, Ferland HL III, Chandramouli A, Owusu-Ankomah M, Nikolich-Zugich T, Bloom AP, et al.(2013a) Disease modification of breast cancer-induced bone remodeling by cannabinoid 2 receptor agonists. J Bone Miner Res 28:92-107.

Lozano-Ondoua AN, Symons-Liguori AM, and Vanderah TW(2013b) Cancer-induced bone pain: mechanisms and models. Neurosci Lett 557:52-59.

Lozano-Ondoua AN, Wright C, Vardanyan A, King T, Largent-Milnes TM, Nelson M, Jimenez-Andrade JM, Mantyh PW, and Vanderah TW(2010) A cannabinoid 2 receptor agonist attenuates bone cancer-induced pain and bone loss. Life Sci 86:646-653.

Luger NM, Honore P, Sabino MA, Schwei MJ, Rogers SD, Mach DB, Clohisy DR, and Mantyh PW(2001) Osteoprotegerin diminishes advanced bone cancer pain. Cancer Res 61:4038-4047.
Luger NM, Mach DB, Sevcik MA, and Mantyh PW(2005) Bone cancer pain: from model to mechanism to therapy. J Pain Symptom Manage 29 (5 Suppl):S32-S46.

Malan TP Jr., Ibrahim MM, Deng H, Liu Q, Mata HP, Vanderah T, Porreca F, and Makriyannis A(2001) CB2 cannabinoid receptor-mediated peripheral antinociception. Pain 93:239-245

Manzanares J, Julian M, and Carrascosa A(2006) Role of the cannabinoid system in pain control and therapeutic implications for the management of acute and chronic pain episodes. Curr Neuropharmacol 4:239-257.

Mercadante S and Fulfaro F(2007) Management of painful bone metastases. Curr Opin Oncol 19:308-314.

Niphakis MJ, Cognetta AB III, Chang JW, Buczynski MW, Parsons LH, Byrne F, Burston JJ, Chapman V, and Cravatt BF(2013) Evaluation of NHS carbamates as a potent and selective class of endocannabinoid hydrolase inhibitors. ACS Chem Neurosci 4:1322-1332.

Nomura DK, Long JZ, Niessen S, Hoover HS, Ng SW, and Cravatt BF(2010) Monoacylglycerol lipase regulates a fatty acid network that promotes cancer pathogenesis. Cell 140:49-61.

Nomura DK, Morrison BE, Blankman JL, Long JZ, Kinsey SG, Marcondes MC, Ward AM, Hahn YK, Lichtman AH, Conti B, et al.(2011) Endocannabinoid hydrolysis generates brain prostaglandins that promote neuroinflammation. Science 334: 809-813.

Ofek O, Karsak M, Leclerc N, Fogel M, Frenkel B, Wright K, Tam J, Attar-Namdar M, Kram V, Shohami E, et al.(2006) Peripheral cannabinoid receptor, CB2, regulates bone mass. Proc Natl Acad Sci USA 103:696-701.

Ohno-Shosaku T, Tanimura A, Hashimotodani Y, and Kano M(2012) Endocannabinoids and retrograde modulation of synaptic transmission. Neuroscientist 18: 119-132.

Schlosburg JE, Blankman JL, Long JZ, Nomura DK, Pan B, Kinsey SG, Nguyen PT, Ramesh D, Booker L, Burston JJ, et al.(2010) Chronic monoacylglycerol lipase blockade causes functional antagonism of the endocannabinoid system. Nat Neurosci 13:1113-1119.

Slosky LM, BassiriRad NM, Symons AM, Thompson M, Doyle T, Forte BL, Staatz WD, Bui L, Neumann WL, Mantyh PW, et al.(2016) The cystine/glutamate antiporter system xc- drives breast tumor cell glutamate release and cancer-induced bone pain. Pain 157:2605-2616.

Sukhtankar D, Okun A, Chandramouli A, Nelson MA, Vanderah TW, Cress AE, Porreca F, and King T(2011) Inhibition of p38-MAPK signaling pathway attenuates breast cancer induced bone pain and disease progression in a murine model of cancer-induced bone pain. Mol Pain 7:81.

Suplita RL II, Farthing JN, Gutierrez T, and Hohmann AG(2005) Inhibition of fattyacid amide hydrolase enhances cannabinoid stress-induced analgesia: sites of action in the dorsolateral periaqueductal gray and rostral ventromedial medulla. Neuropharmacology 49:1201-1209.

Theiner S, Varbanov HP, Galanski M, Egger AE, Berger W, Heffeter P, and Keppler $\mathrm{BK}(2015)$ Comparative in vitro and in vivo pharmacological investigation of platinum(IV) complexes as novel anticancer drug candidates for oral application. J Biol Inorg Chem 20:89-99.

Vanderah TW, Gardell LR, Burgess SE, Ibrahim M, Dogrul A, Zhong CM, Zhang ET, Malan TP Jr., Ossipov MH, Lai J, et al.(2000) Dynorphin promotes abnormal pain and spinal opioid antinociceptive tolerance. J Neurosci 20:7074-7079.

Walser TC, Rifat S, Ma X, Kundu N, Ward C, Goloubeva O, Johnson MG, Medina JC, Collins TL, and Fulton AM(2006) Antagonism of CXCR3 inhibits lung metastasis in a murine model of metastatic breast cancer. Cancer Res 66:7701-7707.

Whiteside GT, Lee GP, and Valenzano KJ(2007) The role of the cannabinoid CB2 receptor in pain transmission and therapeutic potential of small molecule CB2 receptor agonists. Curr Med Chem 14:917-936.

Wilkerson JL, Gentry KR, Dengler EC, Wallace JA, Kerwin AA, Armijo LM, Kuhn MN, Thakur GA, Makriyannis A, and Milligan ED(2012) Intrathecal cannabilactone $\mathrm{CB}(2) \mathrm{R}$ agonist, AM1710, controls pathological pain and restores basa cytokine levels. Pain 153:1091-1106.

Wilkerson JL, Niphakis M.J, Grim TW, Mustafa MA, Abdullah RA, Poklis JL, Dewey WL, Akbarali H, Banks ML, Wise LE, et al.(2016) The selective monoacylglycerol lipase inhibitor MJN110 produces opioid-sparing effects in a mouse neuropathic pain model. J Pharmacol Exp Ther 357:145-156.

Address correspondence to: T.W. Vanderah, Department of Medical Pharmacology, University of Arizona, P.O. Box 245050, Tucson, AZ 85724. E-mail: vanderah@email.arizona.edu 